\title{
A two-phase model for the narrow line region of NGC 4151
}

\author{
S. Komossa
}

Max-Planck-Institut für extraterrestrische Physik, Postfach 1312, 85741 Garching, Germany

Received 9 February 2001 / Accepted 16 March 2001

\begin{abstract}
NGC 4151 is one of the brightest and best-studied Seyfert galaxies. Here, we present a two-phase model of the narrow-line region (NLR) of NGC 4151. This study is motivated by (i) the fact that the X-ray spectrum of NGC 4151 is among the flattest known for Seyferts, and (ii) the recent Chandra detection of an $\mathrm{X}$-ray narrow-line region in this galaxy. X-ray spectra as flat as that of NGC $4151\left(\Gamma_{\mathrm{x}} \simeq-1.5\right)$ are expected to favor the presence of two gas phases in pressure equilibrium (Krolik et al. 1981). In the present study, we show that a pronounced two-phase equilibrium develops in the extended emission-line region if we use the observed multi-wavelength spectrum of NGC 4151 to ionize the clouds. The material is stable to isobaric perturbations over a wide range of temperatures. We therefore propose that such a condition has arisen in the NLR of NGC 4151, and that it explains the detection of hot, extended X-ray gas which we identify as the NLR-cloud confining medium.
\end{abstract}

Key words. galaxies: Seyfert - galaxies: individual: NGC 4151 - galaxies: nuclei - galaxies: emission lines $\mathrm{X}$-rays: galaxies

\section{Introduction}

\subsection{NGC 4151}

NGC 4151 is the optically brightest Seyfert galaxy of type 1.5. It is very nearby $\left(r \simeq 20 \mathrm{Mpc}\right.$ for $H_{0}=$ $50 \mathrm{~km} \mathrm{~s}^{-1} / \mathrm{Mpc}$ ) and is therefore excellently suited to study in detail the physical processes in the nuclei of active galaxies (AGN). The extended narrow-emission-line region (NLR) of NGC 4151 has a cone-like geometry (Schulz 1988, 1990; Evans et al. 1993; Boksenberg et al. 1995), interpreted as "radiation cone" (Schulz 1988) caused by anisotropic illumination of the NLR. In the context of the unified model of AGN (Antonucci 1993) such a radiation geometry arises from the partial obscuration of a molecular torus or the anisotropic emission of the accretion disk.

NGC 4151 is the most intensely studied AGN across the electromagnetic spectrum (see Schulz 1995; Ulrich 2000 for detailed reviews). The X-ray spectrum of the galaxy is complex in the soft band (e.g., Weaver et al. 1994) and extends to very high energies (e.g., Perotti et al. 1981; Maisack et al. 1993). The powerlaw spectrum has a mean photon index $\Gamma_{\mathrm{x}} \simeq-1.5$ and is significantly flatter than that of other Seyfert galaxies $\left(\left\langle\Gamma_{\mathrm{x}}\right\rangle \simeq-1.9\right)$. Using the Chandra HETG spectrometer, Ogle et al. (2000) recently reported the detection of a high-temperature, narrow-line, X-ray emitting plasma in NGC 4151, confirming earlier Einstein (Elvis et al. 1983) and ROSAT (Morse et al. 1995) observations of extended X-ray

Send offprint requests to: S. Komossa, e-mail: skomossa@xray.mpe.mpg.de emission in this galaxy. The X-ray gas is spatially coincident with the NLR and extended narrow-line region (ENLR). A hot intercloud medium was one of several possible explanations mentioned repeatedly (e.g., Elvis et al. 1983, 1990; Morse et al. 1995; Ogle et al. 2000). It is therefore important to examine if, and under which conditions, a two-phase equilibrium of the NLR and ENLR clouds is possible.

\subsection{Multi-phase cloud models, and motivation of the present study}

Different mechanisms for the confinement of the emissionline clouds in AGN have been studied over the last few decades. The model originally investigated in detail by Krolik et al. (1981; KMT hereafter) is a two-phase model, consisting of cold line-emitting clouds $\left(T \simeq 10^{4} \mathrm{~K}\right)$ in pressure balance with a hot inter-cloud medium $\left(T \simeq 10^{8} \mathrm{~K}\right)$. KMT assumed a relatively flat X-ray spectrum of their input continuum illuminating the clouds. Later it turned out that Seyfert galaxies typically show steeper X-ray spectra and a pressure balance between a cold, photoionization heated, and a hot, Compton heated phase then no longer exists (e.g., Fabian et al. 1986). More recent studies have demonstrated the presence of an additional stable region of intermediate temperature (Reynolds \& Fabian 1995; Komossa \& Fink 1997; Komossa 2001), and the recent discovery of warm absorbers located in that intermediate region has revived the interest in multi-phase cloud models. 


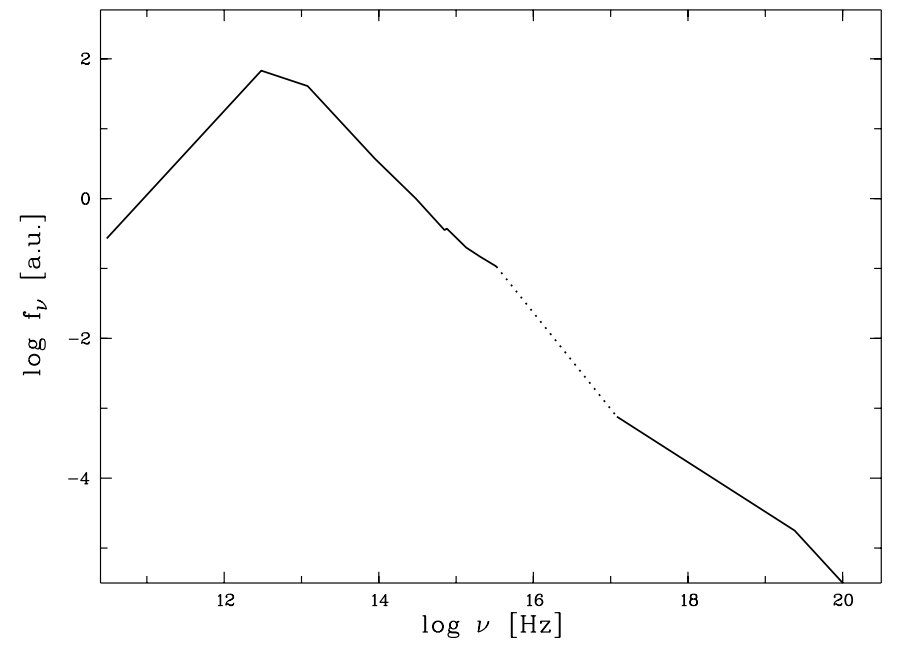

Fig. 1. Average radio to gamma-ray continuum spectrum of NGC 4151, composed from more than 300 individual measurements collected from the literature. The unobserved EUV part of the spectrum is shown as dotted line. The hard X-ray spectrum was conservatively assumed to show a break, whereas most measurements indicated an extent of the spectrum to much higher energies (see Sect. 3.1.1)

The aim of this paper, however, is not a general discussion of the viability of variants of the KMT model to explain the BLR/NLR-confinement in Seyfert galaxies in general. Instead, we address the following question: will a KMT-like two-phase equilibrium develop, if conditions are favorable in individual galaxies?

The observations of extended X-ray emission in NGC 4151 spatially coincident with the NLR, together with the fact that the relatively hard X-ray spectrum of NGC 4151 is favorable for the development of a two-phase equilibrium, motivated the present study.

\section{Model calculations and results}

The stability of photoionized gas clouds to isobaric perturbations can be examined by studying the behavior of cloud temperature $T$ as a function of pressure. If the temperature is multi-valued for constant pressure, and the gradient of the equilibrium curve is positive, several phases may exist in pressure balance.

The photoionization calculations were carried out with the code Cloudy (Ferland 1993) under the following assumptions: we adopted solar metal abundances according to Grevesse \& Anders (1989). The gas density was assumed to be constant $\left(\log n_{\mathrm{H}}=3\right)$, as was the column density $\left(\log N_{\mathrm{H}}=21.5\right)$. Test calculations with different densities and abundances were performed but they do not alter the results discussed here. The clouds were illuminated by the continuum of a central point-like energy source. The spectral energy distribution (SED) used for modeling corresponds to the average multi-wavelength continuum of NGC 4151 (Fig. 1), collected from the literature and determined from more than 300 individual data points (Komossa 1994; Schulz \& Komossa 1993, and references therein). The adopted X-ray spectrum is particularly important in deriving the shape of the equilibrium curve ( $T$ versus $U / T$ ) for high temperatures. We used the mean observed powerlaw index, $\Gamma_{\mathrm{x}}=-1.5$ (e.g., Perotti et al. 1990), extending up to $100 \mathrm{keV}$, where we assumed the spectrum to break steeply into the gamma-ray regime (see Sect. 3.1.1 for further details and references). This mean SED is appropriate for modeling, because the recombination time scale for gas of NLR densities is long, and the NLR thus sees an average continuum.

The cloud temperature as a function of the ionization parameter was extracted after each model run. The ionization parameter is defined as

$U=\frac{Q}{4 \pi r^{2} n_{\mathrm{H}} c}, \quad$ with $Q=4 \pi d^{2} \int_{\nu_{0}}^{\infty} \frac{f_{\nu}}{h \nu} \mathrm{d} \nu$,

where $n_{\mathrm{H}}$ is the gas density, $r$ the distance of the gas cloud from the continuum source, $Q$ the number rate of photons above the Lyman limit $\nu_{0}$, and $d$ the distance between observer and galaxy.

Results are shown in Fig. 2. We find that a clear twophase equilibrium between a low-temperature and a hightemperature component develops over a range of ionization parameters. We have verified that our results are independent of metal abundances ${ }^{1}$, gas density, and the amount of absorbed radiofrequency radiation.

In a next step, we added a black-body component to the UV part of the spectrum. A SED with a huge EUV excess which dominates $Q\left(Q_{\mathrm{bb}} \simeq 0.8 Q_{\text {totl }}\right)$ was invoked by Penston et al. (1990) using the ionization-parametersensitive emission-line ratio $[\mathrm{OII}] /[\mathrm{OIII}]$. This SED would significantly narrow down the region where a two-phase equilibrium is possible (Fig. 2). Since Schulz \& Komossa (1993) showed that the [OII]/[OIII]-ratio overestimates the ionization parameter (thus $Q$ ) if the emission-line region is inhomogeneous in density, and since recent studies did not favor a giant EUV excess in this galaxy (Alexander et al. 1999), we do not discuss this SED further.

For comparison, we have plotted in Fig. 2 the phase diagram obtained using the average SED of a sample of Seyfert galaxies (Komossa \& Schulz 1997). This SED is characterized by a much steeper X-ray spectrum $\left(\Gamma_{\mathrm{x}}=-1.9\right)$ and shows that the temperature of the upper branch has reduced and the overlap between upper an lower branch has disappeared.

\section{Discussion}

\subsection{Model assumptions}

Before we discuss consequences of the models considered here, we first give some cautious comments on the

\footnotetext{
${ }^{1}$ For supersolar metal abundances the small region at $T \simeq$ $10^{6} \mathrm{~K}$ which allows a three-phase equilibrium becomes more prominent. However, photoionization modeling of the ENLR emission-line ratios of NGC 4151 (Schulz \& Komossa 1993) favored depleted metallicity.
} 


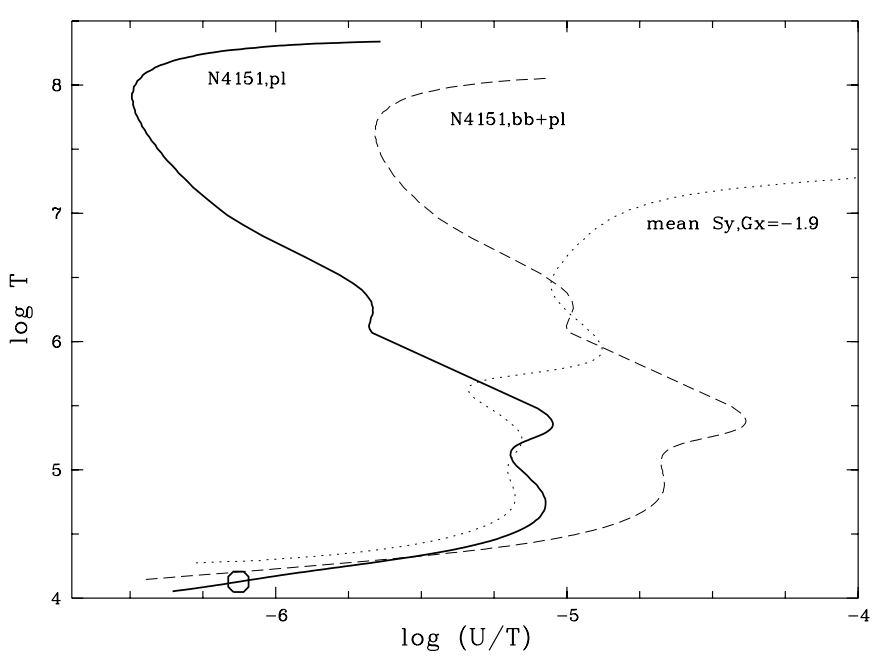

Fig. 2. Equilibrium gas temperature $T$ as a function of $U / T$, obtained using the observed SED of NGC 4151 (thick solid line). The open circle marks a value of $\log U=-2.0$. Two curves are shown for comparison. Dashed line: SED with an additional strong EUV-bump component $\left(Q_{\mathrm{bb}}=0.8 Q_{\text {totl }}\right.$; see text); dotted line: mean Seyfert SED with $\Gamma_{\mathrm{x}}=-1.9$

general model assumptions. The assumptions of photoionization equilibrium and thermal balance have to be kept in mind, as already discussed by KMT. For instance, the gas cooling time scale should be less than the dynamical time scale (Morse et al. 1995 estimate that the time scales are comparable in the ENLR of NGC 4151). The assumption of photoionization equilibrium is problematic if intrinsic source variability and recombination time scale are of the same order. Given the low mean density of the NLR and ENLR of NGC $4151(\log n \simeq 2-3)$ the recombination time scale, $t_{\mathrm{rec}, \mathrm{H}}=\alpha^{-1}\left(T_{\mathrm{e}}\right) n_{\mathrm{e}}^{-1}$, is on the order of $1.310^{2-3} \mathrm{yrs}$, and correspondingly longer for even lower density. This is long compared to the rapid X-ray variability of this source. An average continuum is thus appropriate for modeling. Heating agents in addition to the central continuum source (like cloud friction) were neglected. KMT showed that their influence is generally weak. However, radiation pressure by trapped line photons could significantly increase cloud pressure (Elitzur \& Ferland 1986).

Apart from these general considerations, the influence of the shape of the $E U V-X$-ray continuum specific to $N G C 4151$ on the results is particularly important.

\subsubsection{X-ray continuum shape}

Relevant for the present study is the shape of the Xray spectrum (its steepness, and the position of the break). We used an input spectrum with $\Gamma_{\mathrm{x}}=-1.5$, the mean observed spectral index (e.g., Perotti et al. 1990) ${ }^{2}$, extending up to $100 \mathrm{keV}$. At $100 \mathrm{keV}$ the spectrum shows

\footnotetext{
2 For comparison: the following photon indices (either means over a collection of observations, or single measurements) of the hard X-ray spectrum of NGC 4151 were reported in the
}

a break (Maisack et al. 1993) and continues with a significantly steeper powerlaw. It is interesting to note that early X-ray observations of NGC 4151 detected the source out to much higher energies (e.g., Schönfelder 1978; Perotti et al. 1981; Perotti et al. 1991), whereas some recent observations favored a break at 50-100 keV (Apal'kov et al. 1992; Maisack et al. 1993). Were the early detections of the source based on measurement errors? We do not believe so, because a few early observations did show a break in the spectrum of NGC 4151 (Baity et al. 1984; Bassani et al. 1986). In particular, Baity et al. presented several measurements, of which only one showed a spectral break. Since taken with the same instrument, but at different epochs, measurement errors are unlikely, and it is more plausible that NGC 4151 underwent real spectral variability.

To be on the "safe side", we conservatively assumed the spectrum to break, at $E_{\text {break }}=100 \mathrm{keV}$. Had we adopted a spectrum extending up into the Gamma-regime (MeV energies) the two-phase equilibrium would have become even more pronounced, due to the increased importance of Compton heating.

\subsubsection{EUV spectrum}

Secondly, the EUV spectral shape is of importance for the shape of the equilibrium curve. Using a continuum with a strong EUV excess reduces the Compton temperature of the upper branch and thus narrows down the range were a two-phase equilibrium is possible (Fig. 2; see Fabian et al. 1986; Komossa \& Fink 1997; Komossa \& Meerschweinchen 2000 for details).

Many different EUV continuum shapes of NGC 4151, and the ENLR emission-line response to them, were examined by Schulz \& Komossa (1993). They concluded that the observed ENLR line-ratios can be explained by two very different models: (i) a SED with a huge EUV bump as suggested earlier by Penston et al. (1990) based on $Q$ estimates, and (ii) a powerlaw-like EUV continuum of much lower $Q$, close to the lower limit set by the $\mathrm{H} \beta$ constraint (and a density-mixed, partly matter-bounded ENLR, to produce the same observed emission-line ratios). A continuum of the latter form (ii) was favored by Alexander et al. (1999), and adopted by Kraemer et al. (2000), and we also use it in the present analysis.

\subsection{Two-phase NLR of NGC 4151}

Over the years, a number of different models for the emission-line regions of AGN have been suggested. Most concentrated on the BLR. The broad-line clouds might

literature: Beall et al. (1981): $\Gamma_{\mathrm{x}}=-1.4$, Baity et al. (1984): -1.6, Warwick et al. (1989): -1.54, Maisack \& Yaqoob (1991): -1.37 , Ogle et al. (2000): -1.4 ; for a collection of $\Gamma_{\mathrm{x}}$ measurements of NGC 4151 prior to 1989 see Table 2 of Baity et al. (1984), and Table 2 of Warwick et al. (1989). 
be confined by mechanisms other than thermal pressure (e.g. magnetic fields could play a role; Rees 1987), or they might not be confined at all. Confinement is unnecessary if the BLR clouds are continuously produced (e.g., Perry \& Dyson 1985; Murray \& Chiang 1995). Generally, all suggested models including variants of the KMT approach, still suffer from some unsolved problems.

In the present study, it is not our aim to find the ultimate BLR/NLR model relevant for all types of AGN under any conditions. We rather address the question: will a KMT-like two-phase equilibrium develop in individual galaxies if conditions are favorable? The previous observations of NGC 4151 are highly suggestive that this is indeed the case. Firstly, the X-ray spectrum of NGC 4151 is very hard. Secondly, extended X-ray emission, which could play the role of the NLR/ENLR confining medium, has already been detected. The Chandra observation revealed similar kinematics of optical and X-ray NLR which suggests that they are closely linked (Ogle et al. 2000).

A number of explanations for the hot extended X-ray emission of NGC 4151 have been discussed (Elvis et al. 1983, 1990; Heckman \& Balick 1983; Morse et al. 1995): (i) emission from shocks due to NLR-cloud - ISM interaction. However, this would imply a very small covering fraction of the X-ray gas, and would not work for the ENLR, since cloud velocities (Schulz 1988) are too small. (ii) Scattered nuclear emission was considered a possibility, but Morse et al. (1995) presented estimates which make this scenario unlikely. (iii) Thermal emission from a hot confining medium has been previously mentioned; and we have shown here that a two-phase medium develops if illuminated by the observed SED of NGC 4151.

Some important consistency checks: the mean density of the ENLR, as inferred from the density-sensitive sulphur emission-line ratio, is $\log n=2.3$ (Schulz 1988; Penston et al. 1990). There are indications that the density increases inwards (e.g., Schulz 1995). For the estimates below, we adopt the generally accepted density scaling law of the NLR, of $n \propto \frac{1}{r^{2}}$, with $\log n_{\text {cold }}=2.3$ at $1.5 \mathrm{kpc}$ (corresponding to $\log n_{\text {hot }}=-1.9$; see Fig. 2$)$ and $\log n_{\text {cold }}=6.3$ at $15 \mathrm{kpc}\left(\log n_{\text {hot }}=2.1\right)$. What is the column density of the hot inter-cloud medium? The ENLR of NGC 4151 extends out to $\sim 20^{\prime \prime}(=1.9 \mathrm{kpc})$, similar to the distance to which the X-ray emission has been traced $(1.5 \mathrm{kpc}$, Morse et al. 1995) in the south-west cone. We then expect a column density of $\log N_{\mathrm{H}} \simeq 610^{21} \mathrm{~cm}^{-2}$ of the hot gas. The medium is therefore optically thin to Thomson scattering and would not produce any observable effects which have not been detected. The observed X-ray luminosity of the extended component amounts to $\sim 10^{41} \mathrm{erg} / \mathrm{s}$ (Elvis et al. 1983: $510^{40} \mathrm{erg} / \mathrm{s}$, Morse et al. 1995: $\left.310^{41} \mathrm{erg} / \mathrm{s}\right)$. This compares to an X-ray luminosity of $110^{41} \mathrm{erg} / \mathrm{s}$ predicted by our model, assuming an NLR/ENLR extent and density law as given above, and a total filling factor of the gas that corresponds to a cone opening angle of $75^{\circ}$ (e.g., Evans et al. 1993). We note that this comparison should be regarded an order-of-magnitude estimate, due to uncertainties in both, observations and modelling assumptions. The hot gas phase is highly ionized and we predict the dominance of lines from the ions of, e.g., CVI, NVII, OVIII, SiXIII-XIV, and FeXXIV-XXVI. Ionization stages up to FeXXV have indeed been detected by Chandra.

\section{Summary and conclusions}

The close proximity and brightness of NGC 4151 make it a uniquely valuable object to explore the interaction of the active nucleus with its immediate environment.

We have assessed the influence of the observed flat X-ray continuum of NGC 4151 on the thermal stability of NLR/ENLR clouds and the possibility of cloud confinement by a hot inter-cloud medium. We find that a two-phase medium develops and we suggest to identify the hot phase with the observed extended X-ray emission in NGC 4151, which is co-spatial with the NLR/ENLR.

We estimate a low density of the extended gas which is shown to be able to confine the total NLR and ENLR without becoming Thomson-thick.

The results presented here are relevant for other nearby Seyfert galaxies with relatively hard X-ray spectra (e.g., NGC 3227: $\Gamma_{\mathrm{x}} \approx-1.5$ ). Such galaxies are therefore important targets for future Chandra and XMM-Newton observations.

Acknowledgements. We thank Gary Ferland for providing Cloudy, Hartmut Schulz, Wolfgang Brinkmann and Joachim Trümper for reading the manuscript, and an anonymous referee for useful suggestions and comments. Preprints of this and related papers can be retrieved at http://www. xray.mpe.mpg.de/ skomossa/

\section{References}

Antonucci, R. 1993, ARA\&A, 31, 473

Alexander, T., Sturm, E., Lutz, D., et al. 1999, ApJ, 504, 212

Apal'kov, Y., Babalyan, G., Dekhanov, I., et al. 1992, in Proc: 28th Yamada Conf. on Frontiers of X-ray Astronomy, 28

Baity, W. A., Mushotzky, R., Worral, D. M., et al. 1984, ApJ, 279,555

Bassani, L., Butler, R. C., DiCocco, G., et al. 1986, ApJ, 311, 623

Beall, J. H., Rose, W. K., Dennis, B. R., et al. 1981, ApJ, 247, 458

Boksenberg, A., Catchpole, R. M., Macchetto, F., et al. 1995, ApJ, 440, 151

Elitzur, M., \& Ferland, G. J. 1986, ApJ, 305, 35

Elvis, M., Briel, U., \& Henry, J. P. 1983, ApJ, 268, 105

Elvis, M., Fassnacht, C., Wilson, A. S., \& Briel, U. 1990, ApJ, 361,459

Evans, I. N., Tsetanov, Z., Kriss, G. A., et al. 1993, ApJ, 417, 82

Fabian, A. C., Guilbert, P. W., Arnaud, K. A., et al. 1986, MNRAS, 218, 457

Ferland, G. J. 1993, University of Kentucky, Physics Department, Internal Report

Grevesse, N., \& Anders, E. 1989, in Cosmic Abundances of Matter, ed. C. J. Waddington, AIP 183, 1 (New York: American Institute of Physics) 
Heckman, T. M., \& Balick, B. 1983, ApJ, 268, 102

Komossa, S. 1994, Diploma Thesis, Ruhr-Universität Bochum

Komossa, S. 2001, in Proc: IX. Marcel Grossmann Meeting on General Relativity, Gravitation and Relativistic Field Theories, ed. V. Gurzadyan et al., in press [astro-ph/0101289]

Komossa, S., \& Fink, H. 1997, A\&A, 322, 719

Komossa, S., \& Meerschweinchen, J. 2000, A\&A, 354, 411

Komossa, S., \& Schulz, H. 1997, A\&A, 323, 31

Kraemer, S. B., Crenshaw, D. M., Hutchings, J., et al. 2000, ApJ, 531, 278

Krolik, J. H., McKee, C. F., \& Tarter, C. B. 1981, ApJ, 249, 422

Maisack, M., Johnson, W. N., \& Kinzer, R. L. 1993, ApJ, 407, L61

Maisack, M., \& Yaqoob, T. 1991, A\&A, 249, 25

Morse, J., Wilson, A. S., Elvis, M., \& Weaver, K. 1995, ApJ, 439, 121

Murray, N., \& Chiang, J. 1995, ApJ, 454, L105

Ogle, P. M., Marshall, H. L., Lee, J. C., \& Canizares, C. R. 2000, ApJL, in press [astro-ph/0010314]
Penston, M. V., Robinson, A., Alloin, D., et al. 1990, A\&A, 236,53

Perotti, F., DellaVentura, A., Villa, G., et al. 1981, ApJ, 247, L63

Perotti, F., Buratti, R., Maggioli, P., et al. 1990, ApJ, 356, 467 Perotti, F., Maggioli, P., Quadrini, E., et al. 1991, ApJ, 373, 75

Perry, J. J., \& Dyson, J. E. 1985, MNRAS, 213, 665

Rees, M. J. 1987, MNRAS, 228, 47

Reynolds, C. S., \& Fabian, A. C. 1995, MNRAS, 273, 167

Schönfelder, V. 1978, Nature, 274, 344

Schulz, H. 1988, A\&A, 203, 233

Schulz, H. 1990, AJ, 99, 1442

Schulz, H. 1995, Habilitationsschrift, Ruhr-Universität Bochum

Schulz, H., \& Komossa, S. 1993, A\&A, 278, 29

Ulrich, M.-H. 2000, A\&AR, 10, 134

Warwick, R. S., Yaqoob, T., \& Pounds, K. A. 1989, PASJ, 41, 721

Weaver, K., Mushotzky, R. F., Arnaud, K. A., et al. 1994, ApJ, 423, 621 